\title{
An alternative hypothesis for the outburst mechanism in supergiant fast X-ray transients: the case of IGR J11215-5952
}

\author{
L. Sidoli ${ }^{1}$, P. Romano ${ }^{2,3}$, S. Mereghetti ${ }^{1}$, A. Paizis ${ }^{1}$, S. Vercellone ${ }^{1}$, V. Mangano ${ }^{4}$, and D. Götz ${ }^{5}$ \\ 1 INAF - Istituto di Astrofisica Spaziale e Fisica Cosmica, via E. Bassini 15, 20133 Milano, Italy \\ e-mail: sidoli@iasf-milano.inaf.it \\ 2 INAF - Osservatorio Astronomico di Brera, via E. Bianchi 46, 23807 Merate (LC), Italy \\ 3 Università degli Studi di Milano, Bicocca, Piazza delle Scienze 3, 20126 Milano, Italy \\ 4 INAF - Istituto di Astrofisica Spaziale e Fisica Cosmica, via U. La Malfa 153, 90146 Palermo, Italy \\ 5 CEA-Saclay, DAPNIA/Service d'Astrophysique, 91191 Gif-sur-Yvette Cedex, France
}

Received 22 June 2007 / Accepted 3 October 2007

\section{ABSTRACT}

\begin{abstract}
Context. The physical mechanism responsible for the short outbursts in a recently recognised class of high mass X-ray binaries, the supergiant fast X-ray transients (SFXTs), is still unknown. Two main hypotheses have been proposed to date: the sudden accretion by the compact object of small ejections originating in a clumpy wind from the supergiant donor, or outbursts produced at (or near) the periastron passage in wide and eccentric orbits, to explain the low $\left(\sim 10^{32} \mathrm{erg} \mathrm{s}^{-1}\right)$ quiescent emission. Neither proposed mechanisms explain the whole phenomenology of these sources. IGR J11215-5952, discovered in April 2005 by the INTEGRAL satellite, is a SFXT which undergoes an outburst every 329 days, a periodicity likely associated with the orbital period of the binary system.

Aims. We propose a new explanation for the outburst mechanism, based on the X-ray observations of the unique SFXT known to display periodic outbursts, IGR J11215-5952.

Methods. We performed three Target of Opportunity (ToO) observations with Swift, XMM-Newton and INT EGRAL at the time of the fifth outburst, expected on 2007 February 9. Swift observations of the February 2007 outburst have been reported elsewhere. Another ToO with Swift was performed in July 2007, to monitor the supposed "apastron" passage.

Results. XMM-Newton observed the source on 2007 February 9, for $23 \mathrm{ks}$, at the peak of the outburst, while INT EGRAL started the observation two days later, failing to detect the source, which had already undergone the decaying phase of the fast outburst. XMM-Newton data show large variability, with a bright flare at the beginning of the observation (lasting about $1 \mathrm{~h}$ ), followed by a lower intensity phase (about one order of magnitude fainter) with a large variability as well as low level flaring activity. The spin periodicity discovered by $R X T E$ is confirmed, and a spin-phase spectral variability is observed and studied in detail. The Swift campaign performed in July 2007 reveals a second outburst on 2007 July 24, as bright as that observed about 165 days before. Conclusions. The new X-ray observations allow us to propose an alternative hypothesis for the outburst mechanism in SFXTs, linked to the possible presence of a second wind component, in the form of an equatorial disc from the supergiant donor. We discuss the applicability of the model to the short outburst durations of all other SFXTs, where a clear periodicity in the outbursts has not been found yet. The new outburst from IGR J11215-5952 observed in July suggests that the true orbital period is $\sim 165$ days, instead of 329 days, as previously thought.
\end{abstract}

Key words. X-rays: binaries - stars: neutron - accretion, accretion disks - X-rays: individuals: IGR J11215-5952 - supergiants

\section{Introduction}

The Galactic plane monitoring performed by the INTEGRAL satellite in the last 5 years has allowed the discovery of a number of new high mass X-ray binaries (HMXBs). Several of these new sources are intrinsically highly absorbed and were difficult to discover with previous missions (e.g. IGR J16318-4848, Walter et al. 2003). Others are transient HMXBs (associated with OB supergiant) displaying short outbursts (a few hours, typically less than a day; Sguera et al. 2005), and form the recently recognised new class of supergiant fast X-ray transients (SFXTs).

IGR J11215-5952 is a hard X-ray transient discovered by INT EGRAL during a fast outburst in April 2005 (Lubinski et al. 2005). The short duration of this outburst led Negueruela et al. (2006) to propose that IGR J11215-5952 could be a new member of the class of SFXTs. The optical counterpart is indeed a B-type supergiant, HD 306414 located at a distance of $6.2 \mathrm{kpc}$ (Negueruela et al. 2005; Masetti et al. 2006; Steeghs et al. 2006).
From the analysis of archival INTEGRAL observations and the discovery of two previously unnoticed outbursts, a recurrence period in the X-ray activity of $\sim 330$ days was found (Sidoli et al. 2006, hereafter Paper I), likely linked to the orbital period of the binary system. This periodicity was later confirmed by the fourth outburst from IGR J11215-5952 observed with RXTE/PCA on 2006 March 16-17, 329 days after the previous one (Smith et al. 2006b). The INTEGRAL spectrum was well fitted by a hard power-law with a high energy cut-off around $15 \mathrm{keV}$ (Paper I). Assuming a distance of $6.2 \mathrm{kpc}$, the peak fluxes of the outbursts correspond to a luminosity of $\sim 3 \times 10^{36} \mathrm{erg} \mathrm{s}^{-1}$ (5-100 keV; Paper I). The RXTE/PCA observations showed a possible pulse period of $\sim 195 \mathrm{~s}$ (Smith et al. 2006a), later confirmed during the February 2007 outburst, yielding $P=186.78 \pm$ $0.3 \mathrm{~s}$ (Swank et al. 2007).

All these findings confirmed IGR J11215-5952 as a member of the class of SFXTs, and the first one displaying periodic outbursts. Based on the known periodicity, an outburst 
Table 1. Summary of the XMM-Newton and INTEGRAL pointed observations of IGR J11215-5952.

\begin{tabular}{ccc}
\hline \hline $\begin{array}{c}\text { Start time } \\
\text { (dy mon yr hr:mn:ss) }\end{array}$ & $\begin{array}{c}\text { End time } \\
\text { (dy mon yr hr:mn:ss) }\end{array}$ & $\begin{array}{c}\text { Net exp. } \\
(\mathrm{ks})\end{array}$ \\
\hline $\begin{array}{c}\text { XMM-Newton: } \\
\text { 9 Feb. 2007 16:05:56 }\end{array}$ & 9 Feb. 2007 22:34:16 & 23.3 \\
\hline $\begin{array}{c} \\
\text { INTEGRAL: }\end{array}$ & & \\
11 Feb. 2007 15:07:58 & 12 Feb. 2007 23:12:49 & 111.0 \\
12 Feb. 2007 23:40:07 & 13 Feb. 2007 11:46:59 & 42.0 \\
13 Feb. 2007 12:13:34 & 13 Feb. 2007 19:08:24 & 24.0 \\
13 Feb. 2007 19:41:00 & 14 Feb. 2007 05:11:37 & 33.0 \\
14 Feb. 2007 14:58:35 & 15 Feb. 2007 16:58:23 & 90.0 \\
\hline
\end{tabular}

was expected for 2007 February 9. This allowed us to obtain several Target of Opportunity (ToO) observations with Swift/XRT, XMM-Newton and INTEGRAL. The Swift/XRT results of the February 2007 outburst were reported in Romano et al. 2007 (hereafter Paper II). Here we report the results of the XMM-Newton and INTEGRAL observations of the February 2007 outburst, and of a Swift/XRT campaign performed in July 2007, to monitor the supposed apastron passage.

\section{Observations and data reduction}

\subsection{XMM-Newton data}

IGR J11215-5952 was observed with XMM-Newton on 2007 February 9, with a net exposure of $23.3 \mathrm{ks}$ (see Table 1 for the observations $\log$ ). The observation covered a small fraction of the brightest part of the outburst. In Fig. 1 (upper panel) we show the times of the XMM-Newton and INTEGRAL observations compared with the Swift/XRT simultaneous lightcurve, reported in Paper II.

Data were reprocessed using version 6.5 of the Science Analysis Software (SAS). Known hot, or flickering, pixels and electronic noise were rejected. The response and ancillary matrices were generated using SAS. EPIC PN operated in Small Window mode, while both MOS cameras were in Full Frame mode. MOS and PN observations used the medium thickness filter. Spectra were selected using pattern from 0 to 4 with the $\mathrm{PN}$, and from 0 to 12 with both MOS cameras. Source counts were extracted from circular regions of $40^{\prime \prime}$ radius for the PN and $1^{\prime}$ for MOS1 and MOS2. With the SAS task epatplot we verified that both PN and MOS data were not affected by pileup. Background counts were obtained from similar regions offset from the source position. The background showed evidence for a moderate flaring activity only in the PN, almost at the end of the observation, so we further selected PN in order to exclude this small portion of the observation. No further time selection has been applied to data from both MOS cameras.

To ensure applicability of the $\chi^{2}$ statistics, the net spectra were rebinned such that at least 20 counts per bin were present and such that the energy resolution was not over-sampled by more than a factor 3 . All spectral uncertainties and upper-limits are given at $90 \%$ confidence for one interesting parameter.

\subsection{INTEGRAL data}

The INTEGRAL observations were performed about 2 days after the XMM-Newton observations, i.e. from 2007 February 11 to February 15, and consist of a total of 101 pointings $(\sim 2900 \mathrm{~s}$ each, see Table 1 for details). Version 6.0 of the Off-line Scientific Analysis (OSA) software has been used to analyse
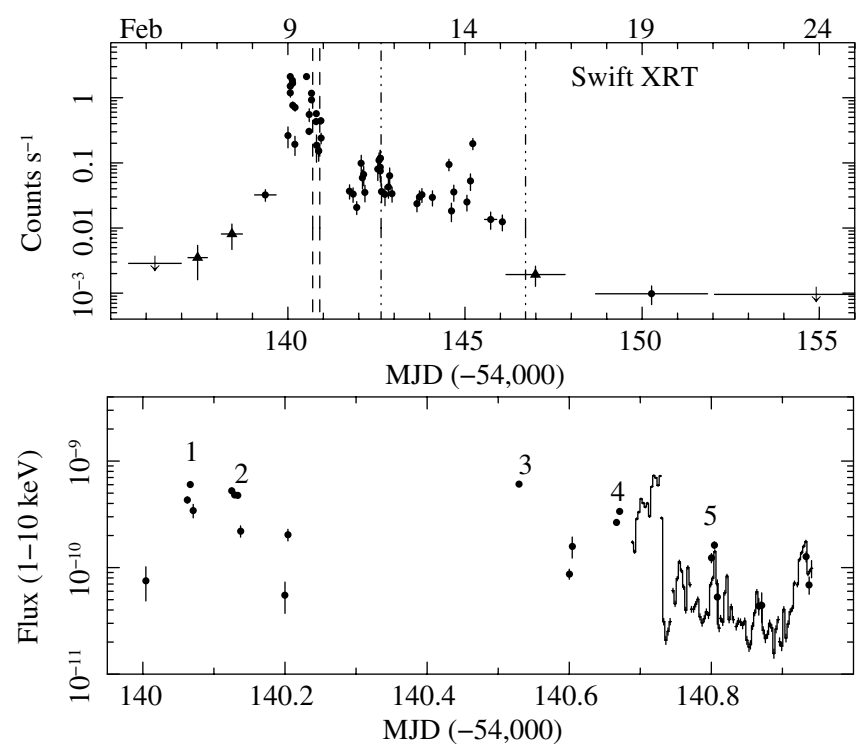

Fig. 1. Upper panel: Swift/XRT lightcurve of the February 2007 outburst from IGR J11215-5952 (from Paper II) together with the start and end times of the XMM-Newton (dashed vertical lines) and of the INTEGRAL (dashed-dotted vertical lines) observations (see Table 1 for details). Day 140 corresponds to 2007 February 9. Lower panel: close-up view of the brightest part of the IGR J11215-5952 lightcurve observed with Swift/XRT (solid circles, 1-10 keV), on 2007 February 9 (adapted from Paper II) together with the XMM-Newton lightcurve (solid line, PN data, $1-10 \mathrm{keV}$ ). The flux is corrected for the absorption and in units of erg cm $\mathrm{cm}^{-2} \mathrm{~s}^{-1}$ in the $1-10 \mathrm{keV}$ range, obtained assuming a power-law spectrum with photon index of 1 and a column density of $10^{22} \mathrm{~cm}^{-2}$ (the numbers mark the "flares" named as in Paper II).

the data. For each pointing, we extracted images in the 22-60 and 60-100 keV energy bands for IBIS/ISGRI and 3-10 and $10-25 \mathrm{keV}$ bands for JEM-X1. For both instruments, the images were used to build a mosaic sky map. Single pointing as well as mosaic results were inspected.

\subsection{Swift data}

Swift observations of the February 2007 outburst have been already discussed in Paper II. The Swift/XRT data reported here were obtained as a ToO campaign aimed at studying the source close to apastron, expected on 2007 July 24. Since IGR J11215-5952 at apastron would have been $<4 \mathrm{~h}$ from the Sun, hence unobservable by Swift under normal circumstances, we spread the $\sim 15 \mathrm{ks}$ estimated to be required for a detection (or firm upper limit) during quiescence over a roughly twomonth time, in short (1.5-2.5 ks) integration times. As a result (see Table 3), IGR J11215-5952 was observed starting on 2007-06-05 17:52 UT through 2007-07-31 10:27 UT, for a total on-source exposure of $16.8 \mathrm{ks}$.

The XRT data were processed with the same standard procedures as described in Paper II (using the Heasoft package, v.6.3.1; spectral redistribution matrices v010).

\section{Analysis and results}

\subsection{February 2007 outburst}

The IGR J11215-5952 lightcurve of the brightest part of the February 2007 outburst, obtained by combining the Swift/XRT and the XMM-Newton PN data, is shown in Fig. 1 (lower 


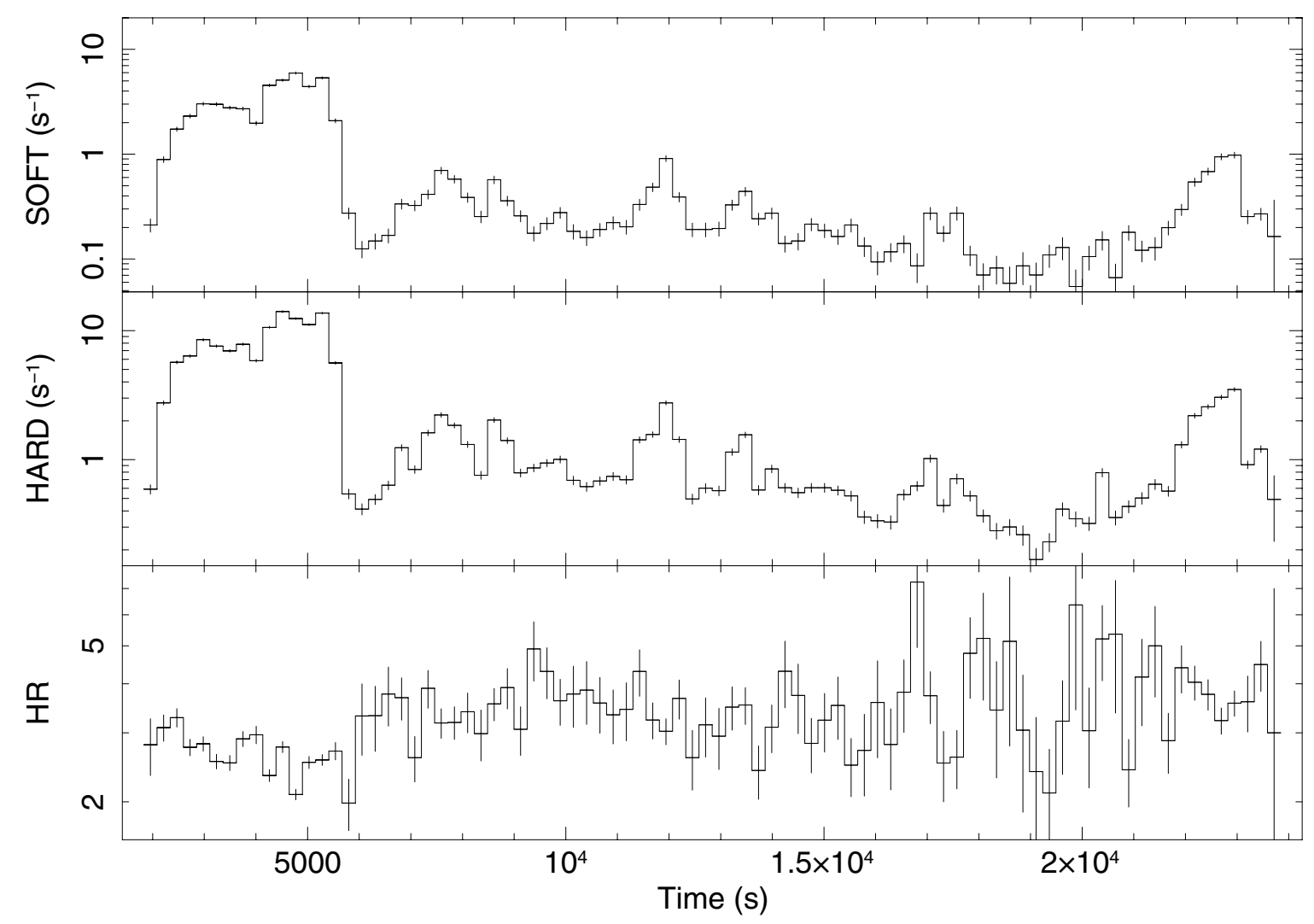

Fig. 2. IGR J11215-5952 background subtracted lightcurve observed with the PN camera, in two energy bands (upper panel, 0.3-2 keV; middle panel, 2-12 keV) together with their hardness ratio (lower panel). A large variability is evident, with a "bright flare" at the beginning of the observation (lasting $\sim 1 \mathrm{~h}$ ), followed by a "faint" phase with a lower count rate level. During this "faint" level of X-ray emission, smaller level flaring activity is also present. Time is in seconds from the beginning of the observation. Bin time is $256 \mathrm{~s}$.

panel). The PN data are shown in more detail in Fig. 2, where the lightcurves in the soft $(0.3-2 \mathrm{keV})$ and hard $(2-12 \mathrm{keV})$ bands and their ratio are displayed. A bright flare is present at the beginning of the observation (which was not caught with Swift/XRT), lasting about $1 \mathrm{~h}$, and displaying large variability (hereafter part "A" of the XMM-Newton observation, from the beginning up to $5500 \mathrm{~s}$ in Fig. 2). Then a lower intensity activity follows (hereafter part "B" of the observation, from about $6000 \mathrm{~s}$ to the end of the observation), also composed by several lower intensity flares.

The hardness ratio as a function of time seems to display some level of variability: fitting it to a constant model yields a reduced $\chi^{2}=3.95$ for 85 degrees of freedom (d.o.f.). Fitting the "A" and "B" parts to a constant we obtain a value of 2.5 and reduced $\chi^{2}=8.22$ (for 13 d.o.f.) for the " $\mathrm{A}$ " part, and a value of 3.3 and reduced $\chi^{2}=1.22$ (for 69 d.o.f.) for the "B" part.

After correcting the arrival times to the Solar System barycenter, we searched for the IGR J11215-5952 spin periodicity by using epoch folding techniques, finding a period of $186.94 \pm 0.58 \mathrm{~s}$, compatible with that derived by XTE/PCA data (Swank et al. 2007).

The folded lightcurves (EPIC PN, background subtracted) in two different energy ranges are shown in Fig. 3, together with their hardness ratios. The pulse profile changes between the "bright" and the "faint" part of the observation. In the bright part ("A") the folded lightcurve shows a double-peaked shape, and the main pulse peaks around phase $0.3-0.4$ (and the secondary peak at 0.8 ), while the "B" lightcurve shows a single broad pulse, around phase 0.0 (where the "A" lightcurve shows a minimum).
We initially extracted two specta, one from the part "A" (bright initial flare), and the second from the part "B" (the fainter second part). Fitting together PN, MOS1 and MOS2 spectra, an absorbed power-law does not fit the data well. Good results were obtained with a cut-off power-law, giving an absorbing column density of $(0.57 \pm 0.03) \times 10^{22} \mathrm{~cm}^{-2}$ ("A") and $(0.7 \pm 0.1) \times$ $10^{22} \mathrm{~cm}^{-2}$ ("B"), a photon index $\Gamma=0.37 \pm 0.09$ ("A") and $\Gamma=$ $0.0 \pm 0.16$ ("B"), a high energy cut-off, of $7 \pm 1 \mathrm{keV}$ ("A") and of $4 \pm 1 \mathrm{keV}$ ("B"; see Fig. 4 for the two count spectra and Fig. 5 for the confidence contour plots).

From the folded lightcurves (Fig. 3), there is evidence that the hardness ratios vary with the spin phase, with lower hardness ratios concentrated in the pulse phase [0.4-0.8] (independent from the source state, "A" or "B"). This suggested that we should perform a phase resolved spectroscopy, extracting spectra for each of the two source intensity states, in two phase intervals: [0.4-0.8] (phase resolved spectra "1", A1 or B1, see Fig. 6) and $(0.8-1.4)$ (phase resolved spectra " 2 ", A2 or B2). It is also evident from the two hardness ratios shown in Fig. 3 that when the source is brighter (state "A"), it is also softer (on average).

An absorbed power-law spectrum is never the best-fit to all the four analysed spectra, leading to structured residuals, particularly evident when fitting the spectra extracted from spin phase (0.8-1.4). This suggested the addition of a soft component (a blackbody), or the use of a power-law with a high energy cut-off. The fit results are equally adequate in the two cases (see Table 2).

An emission line from iron is never required by the data. If we add a Gaussian line at $6.4 \mathrm{keV}$ to the cut-off powerlaw continua of the "bright" spectra, we obtain only a marginal 
A
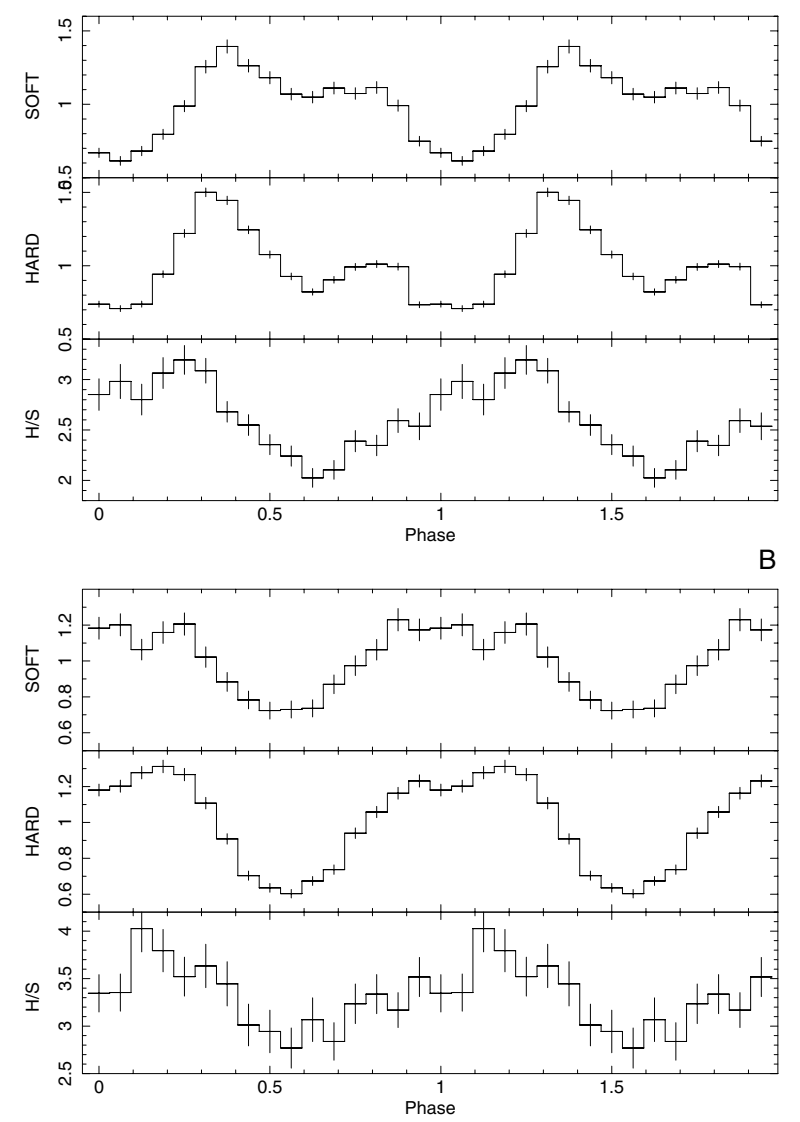

Fig. 3. IGR J11215-5952 lightcurves (normalised intensities) in different energy bands ("soft" means $0.3-2 \mathrm{keV}$, "hard" $2-12 \mathrm{keV}$ ), folded on the period of $187 \mathrm{~s}$, together with their hardness ratios H/S. From top to bottom, "A" is the folded lightcurve of the "bright" part, while " $\mathrm{B}$ " is the folded lightcurve of the "faint" second part of the observation. Note that all lightcurves assume the same starting epoch (MJD = 54 140).

$\left(\sim 3 \sigma\right.$ level) detection with a line flux of $\left(5.7_{-1.8}^{+1.7}\right) \times 10^{-5}$ photons $\mathrm{cm}^{-2} \mathrm{~s}^{-1}$ for phase range [0.4-0.8], and $\left(5.0_{-1.6}^{+2.6}\right) \times$ $10^{-5}$ photons $\mathrm{cm}^{-2} \mathrm{~s}^{-1}$ for phase range $(0.8-1.4)$ (uncertainties in the total line fluxes are $1 \sigma$ ).

Source spectra observed with the RGS arrays (0.3-2.0 keV) resulted in count rates of $(2.02 \pm 0.15) \times 10^{-2} \mathrm{~s}^{-1}$ and $(1.62 \pm$ $0.15) \times 10^{-2} \mathrm{~s}^{-1}$, respectively with RGS1 and RGS2 (22 ks exposure). Since they did not show any evidence for strong emission or absorption lines, we do not discuss them further.

The source is not detected by INTEGRAL either at the single pointing level or in the mosaic. We obtain $3 \sigma$ upper limits of $\sim 1 \mathrm{mCrab}$ and $\sim 5 \mathrm{mCrab}$ in the $22-60$ and $60-100 \mathrm{keV}$ IBIS/ISGRI bands ( $\sim 290 \mathrm{ks})$ and upper limits of $\sim 3 \mathrm{mCrab}$ and $\sim 6 \mathrm{mCrab}$ in the $3-10 \mathrm{keV}$ and $10-25 \mathrm{keV}$ JEM-X1 bands $(\sim 55 \mathrm{ks})$.

These upper limits can be compared with the simultaneous Swift/XRT observations (see Fig. 1, upper panel). The Swift/XRT spectrum resulted in a net exposure time of $18 \mathrm{ks}$. Fitting it with an absorbed power-law, we obtain an absorbing column density of $(1.8 \pm 0.7) \times 10^{22} \mathrm{~cm}^{-2}$ with a photon index of $1.9 \pm 0.5$. The resulting $3-10 \mathrm{keV}$ flux is $1.4 \times$ $10^{-12} \mathrm{erg} \mathrm{cm}^{-2} \mathrm{~s}^{-1}$, more than one order of magnitude below the upper limits derived from JEM-X. The extrapolation of this power-law model to the IBIS/ISGRI energy range results in the following fluxes: $1.5 \times 10^{-12} \mathrm{erg} \mathrm{cm}^{-2} \mathrm{~s}^{-1}(22-60 \mathrm{keV})$ and $8.6 \times 10^{-13} \mathrm{erg} \mathrm{cm}^{-2} \mathrm{~s}^{-1}(60-100 \mathrm{keV})$, which are again more than one order of magnitude lower than the IBIS/ISGRI upper limits.

\subsection{July 2007 outburst}

A new outburst was observed with Swift/XRT in July 2007, during the times of the apastron passage, expected based on the orbital period of 329 days.

The source was not detected in the first seven observations (2007 June 5 to July 17), for which a 3- $\sigma$ upper limit can be placed at $3 \times 10^{-13} \mathrm{erg} \mathrm{cm}^{-2} \mathrm{~s}^{-1}$. On July 24 , the source flux increased above count rates for which pile-up correction is required. Therefore we extracted the source events from an annular region with radii 4 and 30 pixels during the first orbit of data, when the source was piled-up, and from a circular region with a radius of 11 pixels for the rest of the observation. To account for the background, we also extracted events within an annular region centred on the source and with radii 40 and 100 pixels, free from background sources.

Figure 7 shows the background subtracted 1-10 keV light curve, which is corrected for point-spread function (PSF) losses, due to the extraction region geometry, bad/hot pixels, and columns falling within this region, and for vignetting.

The spectrum of the outburst (observation 31) was fit in the 0.5-9 keV energy range, adopting a single power law, with photon index $\Gamma=0.98_{-0.26}^{+0.28}$, and column density of $N_{\mathrm{H}}=$ $\left(1.15_{-0.32}^{+0.42}\right) \times 10^{22} \mathrm{~cm}^{-2}\left(\chi_{\text {red }}^{2}=1.08 / 27\right.$ d.o.f. $)$. These results are fully consistent with those obtained for the 2007 February 9 data (Paper II). The unabsorbed $1-10 \mathrm{keV}$ flux was $\sim 1.1 \times$ $10^{-10} \mathrm{erg} \mathrm{cm}^{-2} \mathrm{~s}^{-1}$.

\section{Discussion}

The observations reported here, together with the Swift/XRT monitoring (Paper II) represent the most complete observation of a SFXT outburst, and demonstrate that the accretion phase in these short transients lasts longer than previously thought on the basis of less sensitive instruments. Indeed, only the brightest part of the outburst, which is short and lasts a few hours, would have been seen by the INTEGRAL instruments. This is clearly demonstrated by the fact that INTEGRAL, starting the observations only 2 days after the onset of the outburst, did not detect the source.

The XMM-Newton observation was performed during the bright part of the IGR J11215-5952 outburst (on February 9), and shows a large intensity variability, which had already emerged during the Swift/XRT observation, but now $X M M$-Newton interestingly fills the gaps in the Swift/XRT observations and a flare which Swift/XRT missed was caught. At least two source states are observed with XMM-Newton with a "bright" flare (lasting $\sim 1 \mathrm{~h}$ ) which reaches a flux at least one order of magnitude larger than the fainter state. Intense flaring activity is observed not only in the bright state, but also in the fainter state, with flares typically lasting a few thousands of seconds.

The spin period found with RXTE (Smith et al. 2006a; Swank et al. 2007) is confirmed and for the first time a pulse profile below $2 \mathrm{keV}$ is reported (see Fig. 3). The hardness ratio is modulated on the pulsar spin period as well. The pulse profiles are different in the two source states, with a double-peaked shape during the bright phase, and a broad single pulse profile during the faint state. 
Table 2. Spin-phase spectroscopy of IGR J11215-5952 (PN+MOS1+MOS2 data), with two models: an absorbed cut-off power-law CUTOFFPL model in XSPEC, and an absorbed power-law together with a blackbody. "A" and "B" spectra refer to the "bright" and "faint" source states, respectively, while " 1 " and " 2 " mean the two spin phase ranges [0.4-0.8] and (0.8-1.4), respectively. $\Gamma$ is the power-law photon index, $E_{c}$ the high energy cut-off in the CUTOFFPL model, $k T_{\mathrm{bb}}$ and $R_{\mathrm{bb}}$ are the temperature and the radius of the blackbody emitter (assuming a distance of $6.2 \mathrm{kpc}$ ); fluxes are corrected for the absorption, in the energy range $0.5-10 \mathrm{keV}$.

\begin{tabular}{|c|c|c|c|c|c|c|c|}
\hline Spectrum & $\begin{array}{c}N_{\mathrm{H}} \\
\left(10^{22} \mathrm{~cm}^{-2}\right)\end{array}$ & $\Gamma$ & $\begin{array}{c}E_{\mathrm{c}} \\
(\mathrm{keV})\end{array}$ & $\begin{array}{l}k T_{\mathrm{bb}} \\
(\mathrm{keV})\end{array}$ & $\begin{array}{l}R_{\mathrm{bb}} \\
(\mathrm{km})\end{array}$ & $\begin{array}{c}\text { Flux } \\
\left(10^{-11} \mathrm{erg} \mathrm{cm}^{-2} \mathrm{~s}^{-1}\right)\end{array}$ & red. $\chi^{2}$ (d.o.f.) \\
\hline \multicolumn{8}{|c|}{ Cut-off powerlaw } \\
\hline A1 & $0.62 \pm 0.04$ & $0.60 \pm 0.11$ & $11_{-2}^{+4}$ & - & - & 6.7 & $1.29(532)$ \\
\hline A2 & $0.56 \pm 0.04$ & $0.25 \pm 0.10$ & $7.3_{-1.0}^{+1.3}$ & - & - & 9.9 & $1.18(589)$ \\
\hline B1 & $0.71 \pm 0.11$ & $0.0 \pm 0.23$ & $4.1_{-0.7}^{+1.1}$ & - & - & 0.5 & 0.99 (427) \\
\hline B2 & $0.79 \pm 0.07$ & $0.0 \pm 0.15$ & $4.8_{-0.6}^{+0.8}$ & - & - & 1.3 & $1.08(574)$ \\
\hline \multicolumn{8}{|c|}{ Powerlaw plus blackbody } \\
\hline $\mathrm{A} 1$ & $0.73_{-0.06}^{+0.07}$ & $1.23_{-0.16}^{+0.22}$ & - & $2.0_{-0.3}^{+0.2}$ & $0.24 \pm 0.03$ & 6.7 & $1.29(531)$ \\
\hline A2 & $0.64 \pm 0.05$ & $0.89_{-0.09}^{+0.11}$ & - & $1.7 \pm 0.2$ & $0.37 \pm 0.05$ & 10 & $1.19(588)$ \\
\hline B1 & $0.8_{-0.2}^{+0.1}$ & $0.96_{-0.39}^{+0.21}$ & - & $1.4 \pm 0.2$ & $0.14_{-0.01}^{+0.06}$ & 0.5 & $1.0(426)$ \\
\hline $\mathrm{B} 2$ & $0.65_{-0.07}^{+0.11}$ & $0.4 \pm 0.3$ & - & $1.3 \pm 0.1$ & $0.27 \pm 0.04$ & 1.3 & $0.99(573)$ \\
\hline
\end{tabular}

Table 3. Swift/XRT observation log.

\begin{tabular}{lllll}
\hline \hline Sequence & Start time (MJD) & $\begin{array}{l}\text { Start time (UT) } \\
\text { (yyyy-mm-dd hh:mm:ss) }\end{array}$ & $\begin{array}{l}\text { End time (UT) }_{\text {(yyyy-mm-dd hh:mm:ss) }} \\
\text { 2007-06-05 21:15:58 }\end{array}$ \\
\hline 00030881024 & 54256.7449 & $2007-06-0517: 52: 35$ & 2007 Net exposure & 1519 \\
00030881025 & 54263.4918 & $2007-06-1211: 48: 11$ & $2007-06-1216: 57: 58$ & 1522 \\
00030881026 & 54270.3808 & $2007-06-1909: 08: 17$ & $2007-06-1911: 05: 57$ & 1843 \\
00030881027 & 54277.1485 & $2007-06-2603: 33: 52$ & $2007-06-2605: 27: 57$ & 2109 \\
00030881028 & 54284.8571 & $2007-07-0320: 34: 14$ & $2007-07-0323: 59: 57$ & 2347 \\
00030969001 & 54294.5627 & $2007-07-1313: 30: 15$ & $2007-07-1407: 10: 56$ & 2126 \\
00030881030 & 54298.0425 & $2007-07-1701: 01: 12$ & $2007-07-1702: 58: 57$ & 2069 \\
00030881031 & 54305.0012 & $2007-07-2400: 01: 42$ & $2007-07-2413: 16: 55$ & 1551 \\
00030881032 & 54312.2343 & $2007-07-3105: 37: 23$ & $2007-07-3110: 26: 56$ & 1695 \\
\hline
\end{tabular}

${ }^{a}$ The exposure time is spread over several snapshots (single continuous pointings at the target) during each observation.


Fig. 4. IGR J11215-5952 count spectra fitted with an absorbed cut-off powerlaw (see text for the parameters): in the left panel the spectra $(\mathrm{PN}+\mathrm{MOS} 1+\mathrm{MOS} 2)$ extracted from the bright initial flare ("A"), while in the right panel the spectra taken from the fainter part of the observation ("B") are shown, together with the residuals (lower panels).

The phase resolved spectra show evidence for a spectral change during the bright flare, where the spectrum is softer during spin-phase range [0.4-0.8], covering the secondary minimum and the rising to the secondary maximum.

We adopted two pheonomenological models to fit the X-ray continua: a cut-off power-law, which is a standard model for this kind of source (e.g. Coburn et al. 2002) and a combination of a power-law plus a blackbody component (with $k T \sim$ $1-2 \mathrm{keV}$ ), also used many times in describing the accreting pulsars X-ray emission (e.g. La Palombara \& Mereghetti 2006). The resulting blackbody radii (a few hundred meters) are consistent with emission in the accretion columns, as proposed and studied in detail by Becker \& Wolff (2005). We note that we successfully fitted the Swift/XRT spectra (Paper II) with the models 


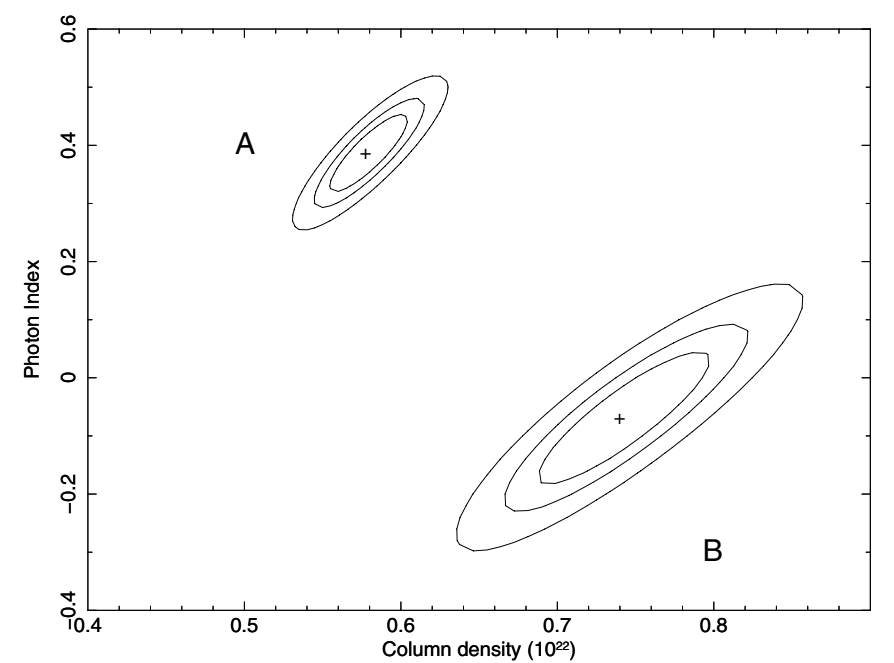

Fig. 5. Confidence contours levels $(68 \%, 90 \%$ and $99 \%)$ for the two parameters of the cut-off power-law model applied to the two intensity selected spectra from the overall "bright" (A) and the "faint" (B) phases.



Fig. 6. Confidence contours levels $(68 \%, 90 \%$ and $99 \%)$ for the two parameters of the cut-off power-law model applied to the four spinphase selected spectra reported in Table 2. Dotted lines refers to the B2 spectrum.

reported here, with similar results. The smaller statistics (and the less extended energy range) of Swift/XRT data compared with $X M M-N e w t o n$, did not require models more complex than an absorbed powerlaw.

The X-ray observations of the IGR J11215-5952 outbursts can be used to test the different models proposed to explain the SFXT's short outbursts. Negueruela et al. (2006) suggested that SFXTs are HMXBs in wide eccentric binaries, to explain the low quiescent luminosity $\left(\sim 10^{32} \mathrm{erg} \mathrm{s}^{-1}\right)$ and the high dynamic range observed $\left(L_{\text {outburst }} / L_{\text {quiescent }}=10^{3}-10^{4}\right)$.

Several authors applied the Bondi-Hoyle accretion to windfed X-ray binaries in eccentric orbits (e.g. Raguzova \& Lipunov 1998 , and references therein). In order to derive the X-ray luminosity variations expected from the motion of the neutron star along the orbit around a supergiant companion, we assume the equations outlined in Reig et al. (2003). In Fig. 8 we compare the Swift/XRT lightcurve with the X-ray emission expected from Bondi-Hoyle accretion onto the neutron star from a spherically symmetric homogeneous wind from a B1-type supergiant in an eccentric orbit. We assumed a beta-law for the supergiant wind, with an exponent $\beta=1$, a stellar mass of $39 M_{\odot}$, a radius of $42 R_{\odot}$, a wind terminal velocity of $1200 \mathrm{~km} \mathrm{~s}^{-1}$ (Lefever et al. 2007) and a wind mass loss of $3.7 \times 10^{-6} M_{\odot} \mathrm{yrs}^{-1}$ (Vink et al. 2000). Note however that mass, radius, wind mass loss and terminal velocity of the supergiant donor are largely uncertain. In any case, for any reasonable choice of the companion wind parameters, the IGR J11215-5952 lightcurve is too narrow and steep to be explained with accretion from a spherically symmetric wind, even adopting extreme eccentricities for the binary system.

The difficulties of such simple models to explain the short duration outbursts in some Be X-ray transients were already noticed (e.g. Stella et al. 1986; or Raguzova \& Lipunov 1998). Centrifugal inhibition of the accretion, occurring when the magnetospheric radius becomes larger than the corotation radius, due to changes in the wind parameters along the eccentric orbit, has been invoked as a possible mechanism at work in these systems. For a slow pulsator such as IGR J11215-5952 and the wind parameters quoted above, the centrifugal inhibition near the periastron passage would require an unusual magnetic field of the order of $10^{14} \mathrm{G}$. However, the spectral cut-off at $15 \mathrm{keV}$ (Paper I) is fully consistent with $B \sim 10^{12} \mathrm{G}$ (Coburn et al. 2002), thus leaving ample room for accretion to never be inhibited.

An alternative viable explanation for the sharpness of the observed X-ray lightcurve could be that in IGR J11215-5952 the supergiant wind is not spherically symmetric. We propose that the supergiant wind has a second component (besides the "polar" spherically symmetric one), in the form of an "equatorial disc", inclined with respect to the orbital plane by an angle $\theta$ (with $\theta=90^{\circ}$ if the disc is perpendicular to the orbital plane). The short outburst is then produced when the neutron star crosses this equatorial disc component, denser and slower than the "polar" wind component. Deviations from spherical symmetry in hot massive star winds are also suggested by optical observations (e.g. Prinja 1990; Prinja et al. 2002) and the presence of equatorial disc components, denser and slower with respect to the polar wind, also results from simulations (ud-Doula et al. 2006).

The thickness " $h$ " of the densest part of this disc originating from the supergiant equatorial region can be calculated from the duration of the brightest part of the outburst (which lasts less than 1 day, time needed for the neutron star to cross it) and the neutron star velocity, $100-200 \mathrm{~km} \mathrm{~s}^{-1}: h \sim(0.8-1.7) \times$ $\sin (\theta) \times 10^{12} \mathrm{~cm}$.

The proposed geometry for the source is sketched in Fig. 9, where four different possibilities are outlined. Case "a" represents a neutron star in an eccentric orbit around the supergiant star with an inclined equatorial disc: this geometry explains the periodic equally spaced outbursts and assumes a certain eccentricity (which is allowed not to be extreme in our model, since the outbursts are mainly produced by the geometry, and not by the high eccentricity). On the other hand, a certain eccentricity should be present $(\sim 0.4)$ in order to reach quiescent X-ray luminosities of the order of $\sim 10^{32} \mathrm{erg} \mathrm{s}^{-1}$, which would not be allowed by the accretion from the polar component of the wind along a circular orbit. We note that it is also possible that the quiescent level from IGR J11215-5952 is much higher than previously observed for other SFXTs (detections during quiescence are still lacking). If this case is the correct geometry for IGR J11215-5952, the true orbital period is $~ 165$ days (see also middle panel in Fig. 10, where an orbital period of 164.5 days has been assumed). If the orbit is circular (case "b"), then 329 days would be the true orbital period and a quiescent level 


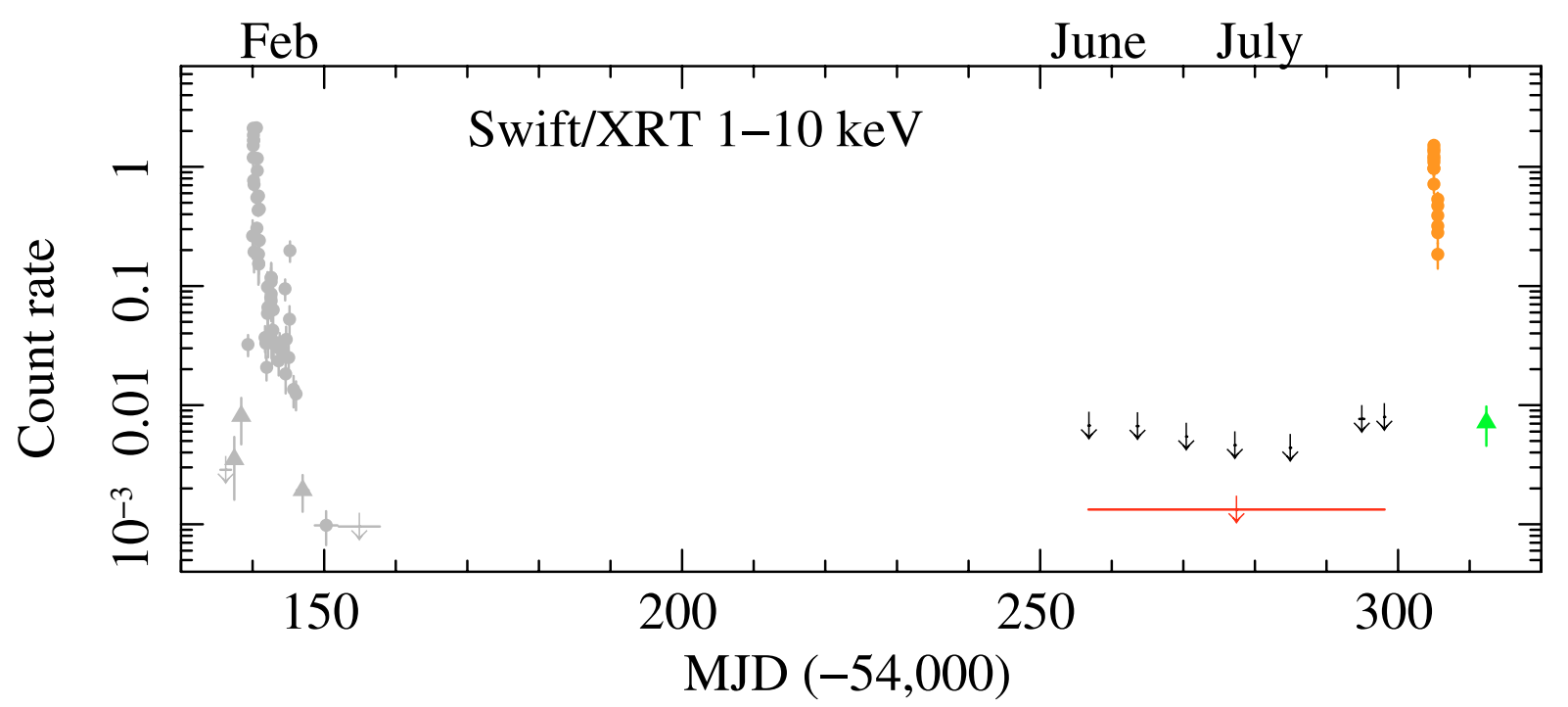

Fig. 7. XRT 1-10 keV light curve, corrected for pile-up, PSF losses, and vignetting and background-subtracted. The grey points were collected during the 2007 February observing campaign (Paper II). The black downward-pointing arrows are 3- $\sigma$ upper limits on single XRT observations (see Table 3) collected between 2007 June 5 and July 17, while the red arrow is the 3- $\sigma$ upper limit obtained by combining them. The remainder of the data (orange and green points) were collected on 2007 July 24 and 31. Different colours denote different observations; filled circles are full detections $(S / N>3)$, triangles marginal detections $(2<S / N<3)$.

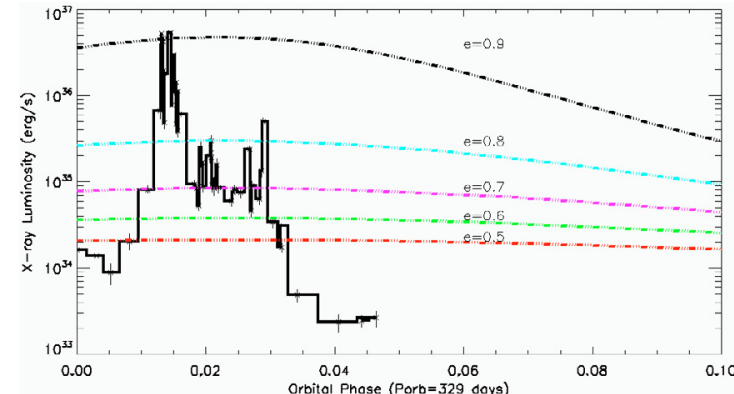

Fig. 8. Comparison of the IGR J11215-5952 lightcurve observed with Swift/XRT with the X-ray emission expected from Bondi-Hoyle accretion from the spherically symmetric wind of the B1-type supergiant companion, for different binary system eccentricities (see text). An orbital period of 329 days is assumed.

of $\sim 10^{33} \mathrm{erg} \mathrm{s}^{-1}$ would be reached. Note however that a lower quiescent emission could be reached also in this $e=0$ case, assuming a much lower mass loss rate from the polar wind component in the B-supergiant. Case " $\mathrm{c}$ " in Fig. 9 is a possibility to explain the short outbursts from all the other SFXTs, where a clear periodicity in the outbursts recurrence has not yet been found. Case " $\mathrm{d}$ " happens when the wind from the equatorial region of the supergiant lies in the orbital plane. This could be the case of the persistent HMXBs (such as Vela X-1). Kaper (1998) found that the observed X-ray luminosities in HMXBs is about one order of magnitude higher than predicted by Bondi-Hoyle accretion assuming standard wind mass loss rates and wind velocities. We suggest that the lower wind velocity and higher density in the form of the proposed equatorial wind could play a role in the observed luminosity, which is higher than predicted.

An accretion disc around the neutron star is unlikely to form, because the wind from the supergiant is too fast. According to Wang (1981) and Waters et al. (1989) the relative velocity of the neutron star with respect to the wind should be less than about $130 \mathrm{~km} \mathrm{~s}^{-1}$ to allow the formation of an accretion disc around the compact object (assuming a neutron star magnetic field of the

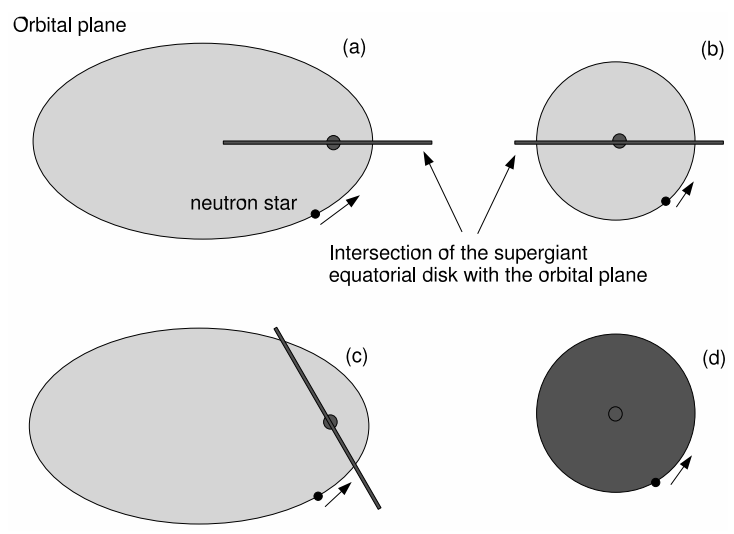

Fig. 9. Proposed geometry for different kinds of systems with supergiant companions. The ellipses (or circles, light colour) mark the orbital plane. The black small circle indicates the neutron star, moving along the orbit. The equatorial wind (in the form of a disc) from the supergiant is inclined with respect to the orbital plane. The dark line sketched here, centred on the supergiant star, marks the intersection of the equatorial wind with the orbital plane. X-ray outbursts occur when the neutron star crosses the inclined equatorial disc from the companion. In case "a" the outburts are periodic and regularly spaced, and a certain eccentricity is required to allow a low quiescent luminosity, which for a circular orbit under the influence of a polar wind would not be reached. Case "b" is also a possibility (circular orbit with inclined equatorial disc and two outbursts per orbit, reaching the same peak flux). Case "c" can be a possibility for all other SFXTs where a clear periodicity in the outbursts has not yet been found. Case "d" sketches the situation where the equatorial disc lies on the orbital plane (this is not the case for SFXTs, but maybe the case for persistent HMXBs, such as Vela $\mathrm{X}-1$ ).

order of $\sim 10^{12} \mathrm{G}$ ). This also excludes the possibility that the fainter X-ray emission in the IGR J11215-5952 lightcurve observed by Swift/XRT after February 9 , is due to the X-ray emission produced from the exhaustion of the matter stored in the accretion disc. To explain this lower level of X-ray activity, we propose a less dense region of the equatorial disc, or a variable density inside the disc. 

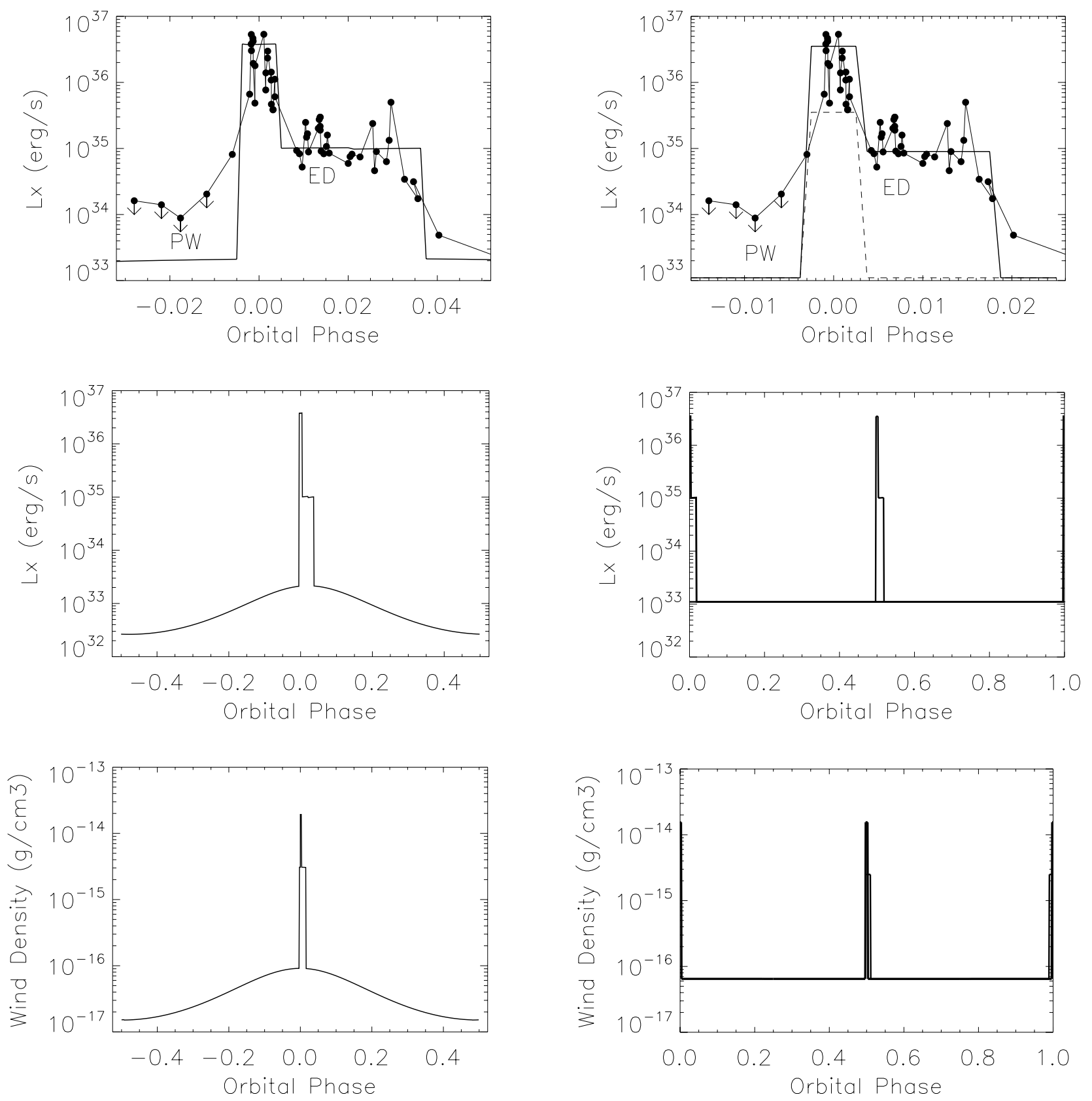

Fig. 10. Results of the proposed model compared with the Swift/XRT lightcurve (upper panels) the expected luminosity variations (middle panels) and wind density along the whole neutron star orbit (lower panels) in two cases: on the left geometry case "a" (with orbital period of 164.5 days and an assumed eccentricity of 0.4) is shown (see Fig. 9), while on the right the results for case "b" are reported (orbital period of 329 days, circular orbit and two similar outbursts per orbit). Both models assume a blue supergiant with a mass of $39 M_{\odot}$ and radius of $42 R_{\odot}$, a "polar wind" component ("PW") with a terminal velocity of $1800 \mathrm{~km} \mathrm{~s}^{-1}$. The X-ray lightcurve observed with Swift/XRT is better reproduced assuming a "polar wind" mass loss rate of $5 \times 10^{-6} M_{\odot} \mathrm{yrs}^{-1}$ for case "a" and $9 \times 10^{-7} M_{\odot} \mathrm{yrs}^{-1}$ for case "b". The second wind component, in the form of an equatorial disc ("ED"), has a variable velocity ranging from $750 \mathrm{~km} \mathrm{~s}^{-1}$ to $1400 \mathrm{~km} \mathrm{~s}^{-1}$ (for case "a"), and from $850 \mathrm{~km} \mathrm{~s}^{-1}$ to $1600 \mathrm{~km} \mathrm{~s}^{-1}$ for case "b". The wind density profiles assumed here are reported in the two lower panels. For a magnetic field of $10^{12} \mathrm{G}$ the centrifugal barrier is open along the entire orbit in both cases. Dashed line in the first upper right figure shows the model prediction based on a ten times smaller wind density with respect to that assumed to reproduce the peak luminosity (maintaining all the other parameters fixed).

In Fig. 10 we show the predictions of our model, compared with the source X-ray lightcurve, with two different sets of orbit and wind parameters, depending on the orbital period assumed for the source. If the orbital period is 329 days, the fact that the two consecutive outbursts observed with Swift reach roughly the same flux at maximum (note however that the data present several gaps so that the maximum might have been missed), implies a circular or a very low eccentricity orbit (as in case "b" in Fig. 9). This case is shown in the right panels in Fig. 10. If instead the period is $\sim 164.5$ days, then the outbursts can be explained with the accretion from the supergiant inclined equatorial disc during the periastron passage, in an eccentric orbit (as in case "a" in Fig. 9).

In the caption of Fig. 10 we report a possible choice for the wind parameters that match the observed X-ray lightcurve. The wind density profile is roughly in agreement with the simulations reported in ud-Doula et al. (2006). For comparison, the winds in the circumstellar discs around main sequence Be stars 
are much denser $\left(10^{-12}-10^{-13} \mathrm{~g} \mathrm{~cm}^{-3}\right.$, e.g. Rinehart et al. 1999). We assume a two-component wind from the companion, with a "polar" spherically symmetric wind ("PW" in Fig. 10, with standard parameters for a B-type supergiant, e.g. Vink et al. 2000; Lefever et al. 2007) together with an inclined equatorial disc ("ED" in Fig. 10) with variable parameters in order to reproduce the variable X-ray emission. Note however that, since the X-ray luminosity produced by the wind accretion is proportional to $\dot{M}_{\mathrm{w}} v_{\text {rel }}{ }^{-4}$ (where $\dot{M}_{\mathrm{w}}$ is the wind mass loss rate, and $v_{\text {rel }}$ is the relative velocity of the wind material with respect to the neutron star, Waters et al. 1989), different combinations of wind density and velocity in the equatorial disc are able to reproduce the $\mathrm{X}$-ray lightcurve as well. Thus, the wind parameters reported in Fig. 10 are only an example.

The high variability with several flares observed both with $X M M-N e w t o n$ and Swift/XRT can be explained by the clumpy nature of the wind in the equatorial disc (as originally proposed by in 't Zand (2005) to explain the outbursts from SFXTs).

The idea that the neutron star intersects an equatorial wind component once or twice during its orbit to explain recurrent flares, is not new in the study of $\mathrm{Be} / \mathrm{X}$-ray binaries (e.g., the Be-transient A0538-66, Apparao 1985). In this respect, the fact that the spin and orbital periods ( $\sim 329$ days or, more likely, a half of this) place IGR J11215-5952 inside the locus typical for Be/X-ray transients of the Corbet diagram (Corbet 1986) is puzzling, since the IGR J11215-5952 donor star is a confirmed B-type supergiant, and not a Be star. Moreover, the source outburst duration appears significantly shorter than that typically displayed by $\mathrm{Be} / \mathrm{X}$-ray binaries. On the other hand, there are other exceptions: for example, the X-ray binary SAX J2103.5+4545, optically identified with a Be star, lies in the region of the Corbet diagram proper for supergiant wind-fed HMXBs (Baykal et al. 2007).

\section{Conclusions}

We have proposed here a new explanation for the short outbursts in SFXTs, based on our results of a monitoring campain of IGR J11215-5952. An equatorial wind from the supergiant companion is suggested, based on the narrow and steep shape of the X-ray lightcurve observed during the latest outburst. The short outburst is suggestive of a deviation from coplanarity $(\theta>$ 0 ) of the equatorial plane of the companion with the orbital plane (e.g., in PSR B1259-63, Wex et al. 1998 or in PSR J0045-7319, Kaspi et al. 1994), and of a some degree of eccentricity $(e>0)$.

Both orbital eccentricity and no-coplanarity can be explained by a substantial supernova "kick" (e.g. Eggleton \& Kiseleva-Eggleton 2001) at birth. This could suggest that SFXTs are likely young systems, probably younger than persistent HMXBs.

We are aware that the derived quantities for the proposed supergiant equatorial disc are highly uncertain and still speculative. This uncertainty clearly emerges from the fact that the observed X-ray lightcurve can be reproduced with both orbital periods (329 days and 164.5 days), different eccentricities and different wind properties. A determination of the orbital eccentricity of the system, for example, would be essential in better constraining the expected properties of the wind which match with the level of X-ray emission during the outbursts. These considerations highlight the need for an as complete as possible deep monitoring of the X-ray emission along the orbit, together with optical observations in order to constrain the supergiant wind properties.

Acknowledgements. Based on observations obtained with XMM-Newton, an ESA science mission with instruments and contributions directly funded by ESA member states and the USA (NASA). Based on observations with INTEGRAL, an ESA project with instruments and the science data centre funded by ESA member states (especially the PI countries: Denmark, France, Germany, Italy, Switzerland, Spain), Czech Republic and Poland, and with the participation of Russia and the USA. We thank the XMM-Newton, INTEGRAL, and Swift teams for making these observations possible, in particular the duty scientists and science planners. L.S. would like to thank all the participants at a seminar at INAF/IASF-Rome, on 2nd March, 2007, which raised very interesting discussions about the source nature, in particular A. Bazzano, P. Persi, V. F. Polcaro and P. Ubertini. P.R. thanks INAF-IASFMi for their kind hospitality. D.G. acknowledges the French Space Agency (CNES) for financial support. This work was supported by contract ASI/INAF I/023/05/0.

\section{References}

Apparao, K. M. V. 1985, ApJ, 292, 257

Baykal, A., Inam, S. Ç., Stark, M. J., et al. 2007, MNRAS, 374, 1108 Becker, P. A., \& Wolff, M. T. 2005, ApJ, 630, 465

Coburn, W., Heindl, W. A., Rothschild, R. E., et al. 2002, ApJ, 580, 394

Corbet, R. H. D. 1986, MNRAS, 220, 1047

Eggleton, P. P., \& Kiseleva-Eggleton, L. 2001, ApJ, 562, 1012

in 't Zand, J. J. M. 2005, A\&A, 441, L1

Kaper, L. 1998, in Properties of Hot Luminous Stars, ed. I. Howarth, ASP Conf. Ser., 131, 427

Kaspi, V. M., Johnston, S., Bell, J. F., et al. 1994, ApJ, 423, L43

La Palombara, N., \& Mereghetti, S. 2006, A\&A, 455, 283

Lefever, K., Puls, J., \& Aerts, C. 2007, A\&A, 463, 1093

Lubinski, P., Bel, M. G., von Kienlin, A., et al. 2005, The Astronomer's Telegram, 469

Masetti, N., Pretorius, M. L., Palazzi, E., et al. 2006, A\&A, 449, 1139

Negueruela, I., Smith, D. M., \& Chaty, S. 2005, The Astronomer's Telegram, 470

Negueruela, I., Smith, D. M., Reig, P., Chaty, S., \& Torrejón, J. M. 2006, in Proceedings of the The X-ray Universe 2005, 26-30 September 2005, El Escorial, Madrid, Spain, ed. A. Wilson (Noordwijk: ESA Publications Division), ESA SP-604, 1, 165

Prinja, R. K. 1990, A\&A, 232, 119

Prinja, R. K., Massa, D., \& Fullerton, A. W. 2002, A\&A, 388, 587

Raguzova, N. V., \& Lipunov, V. M. 1998, A\&A, 340, 85

Reig, P., Ribó, M., Paredes, J. M., \& Martí, J. 2003, A\&A, 405, 285

Rinehart, S. A., Houck, J. R., \& Smith, J. D. 1999, AJ, 118, 2974

Romano, P., Sidoli, L., Mangano, V., Mereghetti, S., \& Cusumano, G. 2007, A\&A, 469, L5

Sguera, V., Barlow, E. J., Bird, A. J., et al. 2005, A\&A, 444, 221

Sidoli, L., Paizis, A., \& Mereghetti, S. 2006, A\&A, 450, L9

Smith, D. M., Bezayiff, N., \& Negueruela, I. 2006a, The Astronomer's Telegram, 773

Smith, D. M., Bezayiff, N., \& Negueruela, I. 2006b, The Astronomer's Telegram, 766

Steeghs, D., Torres, M. A. P., \& Jonker, P. G. 2006, The Astronomer's Telegram, 768

Stella, L., White, N. E., \& Rosner, R. 1986, ApJ, 308, 669

Swank, J. H., Smith, D. M., \& Markwardt, C. B. 2007, The Astronomer's Telegram, 999

ud-Doula, A., Townsend, R. H. D., \& Owocki, S. P. 2006, ApJ, 640, L191

Vink, J. S., de Koter, A., \& Lamers, H. J. G. L. M. 2000, A\&A, 362, 295

Walter, R., Rodriguez, J., Foschini, L., et al. 2003, A\&A, 411, L427

Wang, Y.-M. 1981, A\&A, 102, 36

Waters, L. B. F. M., de Martino, D., Habets, G. M. H. J., \& Taylor, A. R. 1989, A\&A, 223, 207

Wex, N., Johnston, S., Manchester, R. N., et al. 1998, MNRAS, 298, 997 